\title{
MicroRNA-590 is an EMT-suppressive microRNA involved in the TGF $\beta$ signaling pathway
}

\author{
TIANMING LIU*, FANG NIE* ${ }^{*}$ XIANGGUI YANG, XIAOYAN WANG, YUE YUAN, ZHONGSHI LV, \\ LI ZHOU, RUI PENG, DONGSHENG NI, YUPING GU, QIN ZHOU and YAGUANG WENG

\begin{abstract}
Key Laboratory of Laboratory Medical Diagnostics Designated by Chinese Ministry of Education, College of Laboratory Medicine, Chongqing Medical University, Chongqing 400016, P.R. China
\end{abstract}

Received November 26, 2014; Accepted September 9, 2015

DOI: $10.3892 / \mathrm{mmr} .2015 .4374$

\begin{abstract}
Over the last few decades, the epithelial-to-mesenchymal transition (EMT) has been identified as being involved in a number of aspects of physiological processes and various pathological events, including embryonic development and renal fibrosis. Transforming growth factor- $\beta$ receptor 2 (TGF $\beta$ R2) is a widely studied gene, which fulfils a vital role in the TGF $\beta$ signaling pathway and exerts a crucial function in the progression of EMT. Previous studies demonstrated that the dysregulation of microRNAs (miRNAs) is considered to be associated with the EMT process. However, the precise functional involvement of miRNAs in EMT remains to be fully elucidated. In the present study, the level of miR-590 was decreased in an EMT model in vitro and in vivo. Furthermore, the overexpression of miR-590 inhibited EMT by upregulating the epithelial marker, E-cadherin, and downregulating the mesenchymal markers, laminin, $\alpha$-smooth muscle actin $(\alpha$-SMA) and collagen, in the human kidney 2 (HK2) cell line. Furthermore, TGF $\beta$ R 2 was negatively regulated by miR-590. In addition, performing a knockdown of TGF $\beta R 2$ with small-interfering RNA had an effect similar to miR-590 on EMT in the HK2 cell line, whereas the transfection of pCMV-tag2B-TGF $\beta$ R2 reversed the effect of miR-590 on EMT in HK2 cells. Taken together, the present study demonstrated that miR-590 is a novel EMT-suppressive microRNA, which targets TGF $\beta$ R2.
\end{abstract}

Correspondence to: Professor Yaguang Weng, Key Laboratory of Laboratory Medical Diagnostics Designated by Chinese Ministry of Education, College of Laboratory Medicine, Chongqing Medical University, 1 Yixueyuan Road, Yuzhong, Chongqing 400016, P.R. China

E-mail: yaguangweng@126.com

*Contributed equally

Key words: miR-590, epithelial-to-mesenchymal transition, Transforming growth factor- $\beta$ receptor 2, unilateral ureteral obstruction, kidney, HK2

\section{Introduction}

The epithelial-to-mesenchymal transition (EMT) is considered to be an essential biological process, which comprises a series of epithelial plasticity alterations between the epithelial and mesenchymal states, and has an important role in physiological and pathological processes $(1,2)$. During the progression of EMT, epithelial cells, which form monolayers that exist in body tissues and organs, lose their cell-cell adhesiveness, attain an elongated morphology and exhibit an increased motility and invasiveness (1). In addition to these phenotypic changes, the cells may also exhibit alterations in their gene expression, including an upregulation of a variety of transcription factors, including Snail, a downregulation of epithelial markers, including E-cadherin, and an increased expression of mesenchymal markers, such as $\alpha$-smooth muscle actin ( $\alpha$-SMA) (1). Concomitantly with the changes observed in the EMT markers, the further progression through the stages of EMT is marked by the stimulation of epithelial cells, which are transformed into myofibroblasts. EMT is considered to contribute to fibrogenesis by providing a source of myofibroblasts, and also by activating a paracrine signaling pathway between the epithelial and stromal cells (3-5). Furthermore, a number of reviews reported on the role of EMT in epithelial-mesenchymal interactions in the development of fibrotic diseases $(6,7)$. These findings provide substantial evidence for an important role for the epithelium in fibrogenesis, as a source of myofibroblasts, and as a mediator influencing the development of myofibroblasts from epithelia cells.

MicroRNAS (miRNAs) form a class of small, single-stranded, non-coding RNAs, which are 19-22 nucleotides in length and negatively regulate gene expression by base-pairing with the 3' untranslated region (UTR) of cognate mRNAs, which have the conserved seed sequence (8). These non-coding RNAs are considered to exert a critical role in physiological and pathological processes, ranging from embryonic development to tumorigenesis, depending on their specific gene targets $(9,10)$. An accumulating body of evidence supports that miRNAs exert a crucial role in EMT through the modulation of EMT-associated genes. In the cell model of EMT induced by transforming growth factor- $\beta$ (TGF $\beta 1$ ), previous studies have demonstrated that all five members of the miR-200 family and miR-205 are capable of repressing EMT 
by targeting the transcription factors, zinc finger E-box binding homeobox 1 and 2 (ZEB1/2) (11-13). A further study revealed that miR-155 exerts an essential role in TGF $\beta 1$-induced EMT through targeting the protein, RhoA (14). Taken together, these results suggested that miRNAs may be crucially important in EMT, however, the biological functions of the majority of the miRNAs in EMT remain to be fully elucidated.

In the present study, ample evidence is provided to support a role for miR-590 in EMT, based on studies performed in TGF $\beta 1$-treated human kidney 2 (HK2) cells and a unilateral ureteral obstruction (UUO) kidney model. The study aimed to investigate whether the overexpression of miR-590 exerted any influence on EMT in HK2 cells and in human renal tubular epithelial cells. Furthermore, the results of the present study are discussed with respect to potential effects on epithelium homeostasis, and the consequences of these effects in kidney fibrosis.

\section{Materials and methods}

Prediction of miRNAs which target TGF $\beta$ receptor $2(R 2)$. The miRNAs targeting TGF $\beta$ R 2 were predicted using the prediction programs: miRDB (http://www.microrna.org/), PicTar(http:// pictar.mdc-berlin.de/) and TargetScan (http://www.targetscan. org/). Only those miRNAs that were predicted by all three algorithms were selected as putative regulators of TGF $\beta R 2$, and were suggested for further experimental identification.

Cell culture and transfection. Human embryonic kidney 293 (HEK293T) and HK2 cells were cultivated in Dulbecco's modified Eagle's medium and RPMI-1640 media (Invitrogen Life Technologies, Carlsbad, CA, USA), respectively, supplemented with $10 \%$ fetal bovine serum (Invitrogen Life Technologies), $100 \mathrm{U} / \mathrm{ml}$ penicillin and $100 \mu \mathrm{g} / \mathrm{ml}$ streptomycin at $37^{\circ} \mathrm{C}$ in an atmosphere of $5 \% \mathrm{CO}_{2}$. The HEK293T and the HK2 cells were purchased from the American Tissue Culture Collection (Manassas, VA, USA). For the transfection experiments, when the cells had reached $60 \%$ confluence, the HK2 cells were transfected with pCDNA3.1 (800 ng), pCDNA3.1-mir-590 (800 ng), small-interfering (si) RNA-TGF $\beta$ R2 (20 nM; sense and antisense sequences, 5'-GUGCCUGUAACAUGGAAGATT-3' and 5'-UCUUCC AUGUUACAGGCACTT-3', respectively) or siRNA-control (20 nM; sense and antisense sequences, 5'-UUCUCCGAA CGUGUCACGUTT-3' and 5'-ACGUGACACGUUCGGAGA ATT-3', respectively) using Lipofectamine ${ }^{\mathrm{TM}} 2000$ transfection reagent (Invitrogen Life Technologies), according to the manufacturer's instructions.

Plasmid construction. To overexpress the miR-590, the 81 base pairs (bp) genomic sequence, which encodes mature miR-590, was cloned into the PUC57 vector (manufactured by Genewiz, Inc., South Plainfield, NJ, USA). The sequence used was: 5'-AGTCAGAAATGAGCTTATTCATAAAAGTGCAGT ATGGTGAAGTCAATCTGTAATTTTATGTATAAGCTA GTCTCTGATTGA-3'. Subsequently, the premature sequence was transferred into pcDNA3.1 (Invitrogen Life Technologies) downstream of the cytomegalovirus (CMV) promoter between the restriction sites HindIII and EcoRI. For the luciferase reporter plasmids, the $3^{\prime}$ UTR of the mouse TGF $\beta R 2$, or its mutant variations, was amplified by reverse transcription-quantitative polymerase chain reaction (RT-qPCR) from the mouse genome, and inserted downstream of the luciferase coding sequence of the pcDNA3.1-luciferase reporter plasmid between the restriction sites BamHI and EcoRI. For the overexpression of TGF $\beta$ R2, the coding sequence was cloned to generate the plasmid pCMV-tag-2B-TGF $\beta$ R2 using the restriction sites of BamHI and Xhol, and (forward: 5'-CGCGGATCCCTGTCC ACTTGCGACAACCAG-3' and reverse: 5'-GGCCTCGAG TTTGGTAGTGTTCAGCGAGC-3'). All the plasmids used in the present study were verified by sequencing.

Generation of the EMT model in vitro. EMT was induced in the HK 2 cells in the presence of TGF $\beta 1$. Cells that had reached $50 \%$ confluence were treated with recombinant human TGF $\beta 1$ (10 ng/ml; R\&D Systems, Minneapolis, MN, USA) in RPMI-1640 medium for $48 \mathrm{~h}$.

Generation of the UUO kidney model. The UUO kidney model was generated in male C57BL/6 mice (12-16 weeks old, weighing 24-28 g), which were purchased from the Animal Center of Chongqing Medical University. Animals were treated according to the recommendations in the Guide for the Care and Use of Laboratory Animals of the National Institutes of Health. The experimental procedures were approved by the Animal Experimental Ethics Committee of Chongqing Medical University. The animals were operated on by left ureteral ligation, as described previously $(15,16)$. Briefly, following anesthetization with sodium pentobarbital $(50 \mathrm{mg} / \mathrm{kg})$, the middle portion of the left ureter was ligated completely by surgical suture, whereas the control group underwent an identical process without ligation. The mice were sacrificed on day 14 following the ligation surgery, and the kidneys were harvested for subsequent studies. These experiments were approved by the Medicine Animal Care Committee of the Chongqing Medical University.

Histochemical analysis. The kidneys were dissected, fixed with $10 \%$ neutral formalin for $48 \mathrm{~h}$, and embedded in paraffin. Briefly, tissues were immersed in acetone at $4^{\circ} \mathrm{C}$, transferred to a freezer at $-20^{\circ} \mathrm{C}$, and fixed overnight. Then the tissues were dehydrated in acetone at $4^{\circ} \mathrm{C}$ for $15 \mathrm{~min}$ and in acetone for another $15 \mathrm{~min}$ at room temperature. Thereafter the tissues were cleared twice in methyl benzoate for $15 \mathrm{~min}$, and twice in xylene for $15 \mathrm{~min}$ at room temperature. Finally they were penetrated with paraffin (melting point, $58-60^{\circ} \mathrm{C}$; Junsei Chemical Co., Ltd., Tokyo, Japan) at $60^{\circ} \mathrm{C}$ for $2-3 \mathrm{~h}$ in a vacuum-evaporating embedder and embedded in paraffin. Subsequently, 6-mm thick sections were selected and stained with Masson's stain for the determination of the tubulointerstitial index, as previously described (17-19).

TGF $\beta R 2$ 3' UTR-luciferase reporter assay. The luciferase reporter analyses were performed on HEK293T cells. For the transfection assay, the HEK293T cells were seeded $\left(1 \times 10^{5}\right.$ cells/well $)$ in 24 -well plates. The plasmids pcDNA3.1-Luc-TTGFßR2WT, pcDNA3.1-Luc-TGFßR2Mut and pcDNA3.1-Luc were transfected into HEK293T cells, which stably expressed pcDNA3.1-miR-590 or pcDNA3.1, using Lipofectamine ${ }^{\mathrm{TM}} 2000$ in OptiMEM medium (Thermo 
Fisher, Waltham, MA, USA). At 48 h later, the cells were harvested with PBS, and lysed with radioimmunoprecipitation assay (RIPA) buffer. The dual-luciferase reporter assay system (Promega Corp., Madison, WI, USA) was used for the analysis, and the data are shown as the ratio of luciferase activity normalized against that of Renilla.

$R T$ - $q P C R$. RT-qPCR was used to determine the mRNA levels. The total RNA was isolated from cells or kidneys using Trizol ${ }^{\circledR}$ (Invitrogen Life Technologies), and $5 \mu \mathrm{g}$ total RNA was selected to be reverse-transcribed using the First-Strand cDNA Synthesis kit (Thermo Fisher Scientific) according to the manufacturer's instructions. Aliquots of $0.5 \mu \mathrm{l}$ transcription product served as the template for the qPCR reactions with UltraSYBR mixture (CWBio, Inc., Beijing, China) or UltraSYBR mixture (Takara Bio, Inc., Otsu, Japan). The mRNA expression levels of E-cadherin, $\alpha$-SMA and collagen were normalized to 18S RNA, whereas the expression level of miR-590 was normalized to U6 RNA. Primers for the detection of the miRNAs were as follows. The primers for miR-590 were provided by Ruibo (Guangzhou, China); the U6 primers were 5'-CTCGCTTCGGC AGCACA-3' (forward) and 5'-AACGCTTCACGAATTTGC GT-3' (reverse). The primers for the detection of the mRNAs were as follows: $18 \mathrm{~S}$ primers were 5'-GTAACCCGTTGAAC CCCATT-3' (forward) and 5'-CCATCCAATCGGTAGTAG CG-3' (reverse); the mouse E-cadherin primers, 5'-GTCAAC ACCTACAACGCTGC-3' (forward) and 5'-ACGTGCTTGGGT TGAAGA-3' CA (reverse); the mouse $\alpha$-SMA primers, 5 '-GCT ATGCCCTGCCTCATGC-3' (forward) and 5'-TCACGGACA ATCTCACGCTC-3' (reverse); the mouse collagen primers, 5'-AACTTTGCTTCCCAGATGTCCTATG -3' (forward) and 5'-GCTTCCCCATCATCTCCATTCTTGC-3' (reverse); the human E-cadherin primers, 5'-GGTGCTCTTCCAGGAACC TC -3' (forward) and 5'-GGAAACTCTCTCGGTCCAGC-3' (reverse); the human $\alpha$-SMA primers, 5'-GGGGTGATGGTG GGAATG-3' (forward) and 5'-GCAGGGTGGGATGCTCTT-3' (reverse); and the human collagen primers were 5'-AAGGTG TTGTGCGATGACG-3' (forward) and 5'-TGGTCGGTGGGT GACTCTG-3' (reverse). All the experiments were performed in triplicate. The expression levels were quantified using the $2^{-\Delta \mathrm{Ct}}$ method. $\Delta \mathrm{Ct}$ was calculated either as $\mathrm{Ct}$ (E-cadherin/ $\alpha$-SMA/collagen) - Ct (i8s) or as Ct (miR-590) - Ct (U6).

Protein isolation and western blotting. The HK2 cells were washed three times with $100 \mu 1$ ice-cold PBS buffer and lysed by adding $200 \mu 1$ RIPA buffer/well immediately, with a 30-min incubation period. For the kidney tissue, the kidneys were homogenized in lysis buffer (RIPA) containing $1 \mathrm{mg} / 1$ protease cocktail (BioTool, Jupiter, FL, USA; containing 104 mM AEBSF, $80 \mu \mathrm{M}$ aprotinin, $5 \mathrm{mM}$ bestatin, $1.5 \mathrm{mM} \mathrm{E}-64,2 \mathrm{mM}$ leupeptin, $1.5 \mathrm{mM}$ pepstatin A), prior to sonication Xo-1200D (Heng Long Instrument Co., Ltd., Changzhou, China) at $4^{\circ} \mathrm{C}$ for $60 \mathrm{sec}$. The total protein concentration of each sample was measured using the bicinchoninic acid protein assay with a microplate procedure (Beyotime Institute of Biotechnology, Haimen, China), prior to western blotting. Samples of $30 \mu \mathrm{g}$ total protein were subjected to $12 \%$ SDS-PAGE, and the protein bands were subsequently transferred onto polyvinylidene fluoride membranes (Millipore Corp., Bedford, MA, USA). The membranes were blocked with $5 \%$ non-fat milk in
Tris-buffered saline (TBS)/Tween $20(0.1 \%)$ at room temperature for $1 \mathrm{~h}$. Subsequently, the membranes were immersed in primary antibody solution [rabbit polyclonal anti-E-cadherin, 1:3,000 (20648-1-AP; Proteintech Group Inc., Chicago, IL, USA); rabbit polyclonal anti-laminin, 1:2,500 (sc-20145; Santa Cruz Biotechnology, Inc., Santa Cruz, CA, USA); rabbit polyclonal anti-TGF $\beta$ R2, 1:2,000 (our laboratory); or mouse monoclonal anti- $\beta$-tubulin, 1:5,000 (HC101-01 TransGen, Beijing, China)] at $4^{\circ} \mathrm{C}$ for an overnight incubation. After washing three times with TBS/Tween 20 (0.1\%; 5 min each wash), incubation with horseradish peroxidase-conjugated goat anti-rabbit and mouse secondary antibody (1:5,000, Beijing Zhongshan Jinqiao Biotechnology Co., Ltd., Beijing, China) secondary antibodies was performed at room temperature for $1 \mathrm{~h}$ (1:5,000 dilution). The immunoreactivity was developed using chemiluminescent horseradish peroxidase substrate reagent (Millipore Corp.), and the signal was detected using the Bio-Rad ChemiDoc XRS+ imaging system (Bio-Rad Laboratories, Inc., Hercules, CA, USA). $\beta$-tubulin was used as a protein loading control, and western blot analyses were performed in triplicate.

Immunofluorescent analysis. For the detection of immunofluorescence, HK2 cells were cultured with sterile glass coverslips in six-well plates. The glass slides were treated with $95 \%$ alcohol for $15 \mathrm{~min}$ at room temperature prior to cell culture. Subsequently, the cell coverslips were washed three times with $500 \mu \mathrm{l}$ PBS and permeabilized with $0.1 \%$ Triton X-100 for 30 min. Following blocking with $10 \%$ normal goat serum for $1 \mathrm{~h}$ at room temperature, the cells were incubated with rabbit anti-laminin primary antibody (1:100; Santa Cruz Biotechnology, Inc.) for $3 \mathrm{~h}$ at room temperature. The coverslips were subsequently washed three times with PBS, prior to incubation with fluorescein isothiocyanate-conjugated goat anti-rabbit immunoglubulin $\mathrm{G}$ at room temperature for $1 \mathrm{~h}$. The slides were examined under a Nikon (Melville, NY, USA) Eclipse TE300 fluorescence microscope, and images were captured with a SPOT Diagnostic (Sterling Heights, MI, USA) charged-couple device camera.

Statistical analysis. GraphPad Prism 5 (San Diego, CA, USA) software was used to analyze the experimental data. The statistical differences between groups, including the bar graph of the luciferase assay and the results from the RT-qPCR experiments, were analyzed by one-way analysis of variance. All the experiments were performed at least in triplicate, and the statistical errors of averaged data are presented as the mean \pm standard error of the mean. $\mathrm{P}<0.05$ was considered to indicate a statistically significant difference.

\section{Results}

miR-590 is decreased during EMT in vitro and in vivo. To investigate whether miR-590 has a direct function on EMT, TGF $\beta 1$-induced epithelial cells were used as a cell model, and UUO mice were an animal model, for modulating EMT in vitro and in vivo, respectively. TGF $\beta 1$-induced EMT experiments were performed in HK2 cells according to the method described previously $(20,21)$, whereas the UUO model was generated in mice by left ureter ligation (22). Following treat- 
A

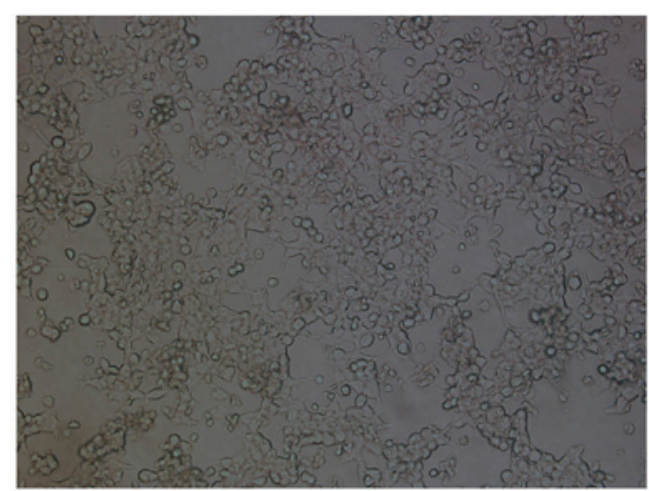

DMSO

B

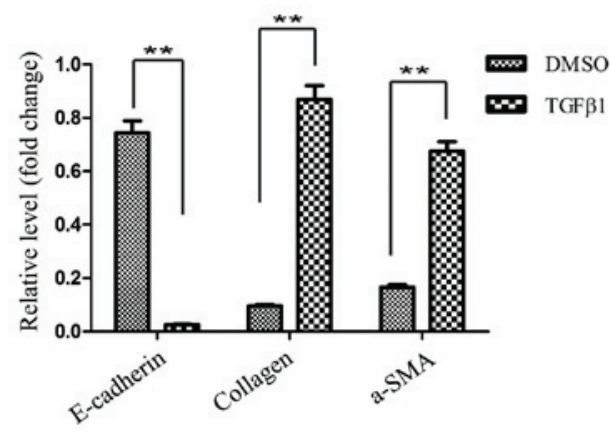

D

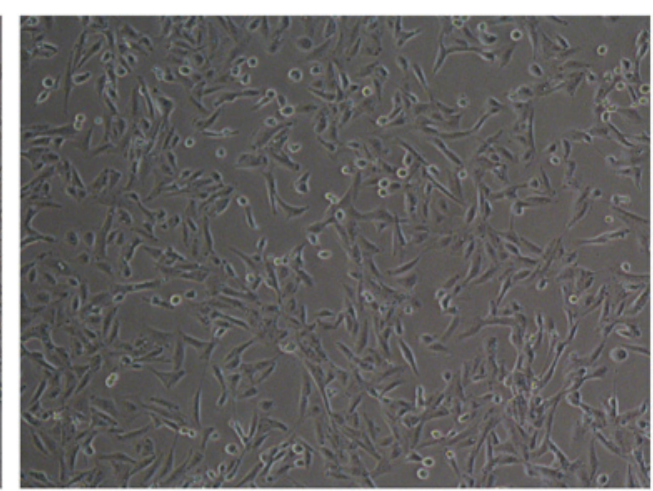

TGF $\beta 1$

C E-cadherin

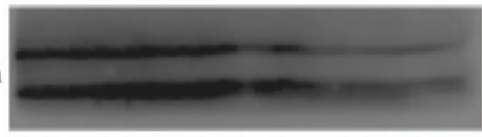

laminin

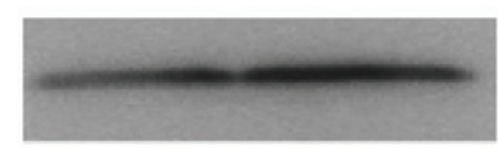

tubulin

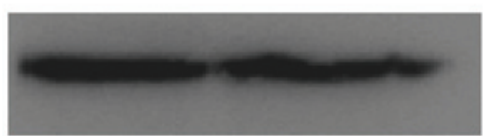

$0^{n S^{O}}$
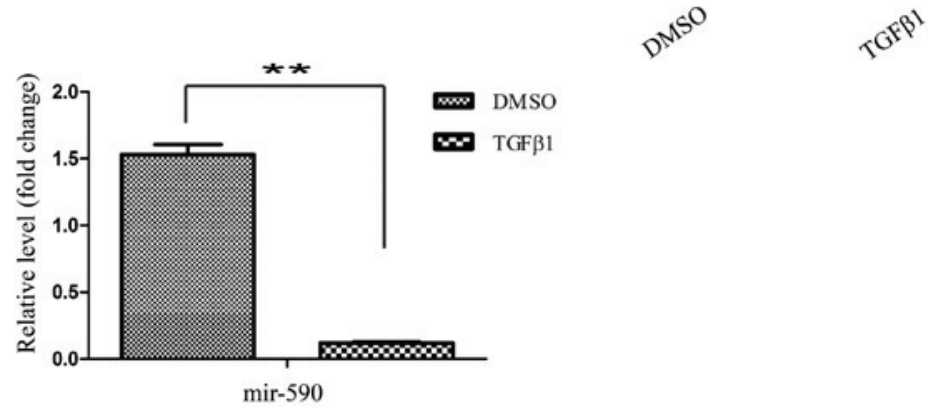

Figure 1. mRNA and protein expression levels of miR-590 were decreased during EMT in vitro. (A) The EMT model with TGF 31 -induced HK2 cells. The morphological changes of the HK2 cells in the presence of TGF $\beta 1$ (10 ng/ml) were observed under a phase-contrast microscope (magnification, x100). (B) The regulation of collagen, $\alpha$-SMA and E-cadherin in the cell EMT model. The mRNA expression levels were detected using RT-qPCR. (C) The regulation of laminin and E-cadherin in the cell EMT model. The protein expression levels were detected by western blotting. (D) The downregulation of miR-590 expression in the cell EMT model. The expression level was examined by RT-qPCR. The results are shown as the fold change compared with the non-treated control. Data are presented as the mean \pm standard deviation of three independent experiments, ${ }^{* *} \mathrm{P}<0.01$ compared with the control. DMSO, dimethyl sulfoxide; TGF $\beta 1$, transforming growth factor $\beta 1$; a-SMA, $\alpha$-smooth muscle actin.

ment with TGF $\beta 1$ for $48 \mathrm{~h}$, the HK2 cells were transformed from the epithelial state, characterized by polygonal morphology and tight connections, into a spindle-like mesenchymal morphology, which appeared as the HK2 cells underwent the process of EMT (Fig. 1A). Subsequently, the EMT process at the molecular level was identified by determining the levels of the marker genes. The results from the RT-qPCR analysis revealed decreased mRNA expression levels of the epithelial marker (E-cadherin), and increased expression levels of the mesenchymal markers, collagen and $\alpha$-SMA, following TGF $\beta 1$ treatment of the HK2 cells (Fig. 1B). Western blot analysis also confirmed the EMT process.

As shown in Fig. 1C, downregulation of the protein expression level of E-cadherin, and an upregulation of the protein expression level of laminin, were observed in TGF $\beta 1$-treated
HK2 cells. Furthermore, the expression level of miR-590 in HK2 cells was significantly decreased following TGF $\beta 1$ treatment, as revealed by the RT-qPCR analysis (Fig. 1D). These results indicated an inverse correlation between the expression of EMT-associated genes and miR-590. To investigate the association between miR-590 and the EMT process in renal fibrosis, the identical experiments were performed in the UUO kidney. Prior to the experiments, the efficiency of the UUO model was confirmed by Masson staining.

The histopathological results revealed substantial structural alterations, induced by left ureter ligation. In the control specimens, the kidney tissues were well aligned and surrounded by only a small quantity of interstitial tissue, whereas the section of the kidney from the UUO-induced mice featured a large proportion of fibres (Fig. 2A). For the EMT analysis, 
A

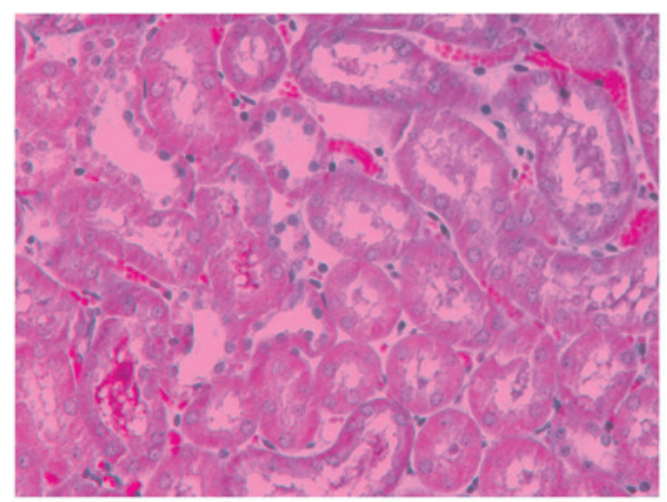

Control

B

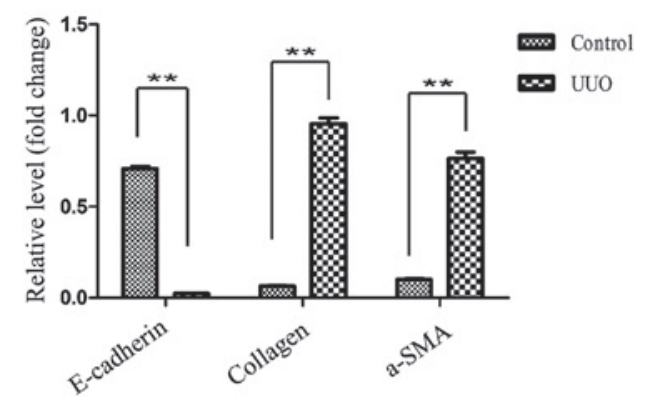

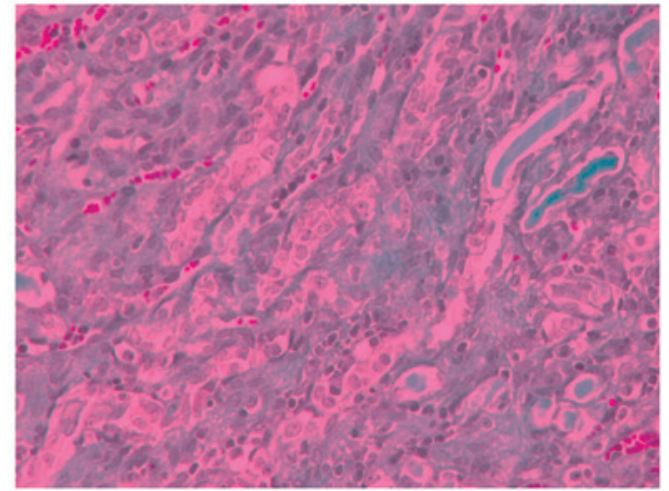

UUO

$\mathbf{C}$

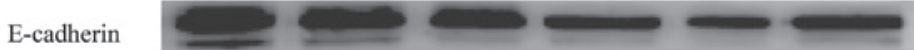

laminin

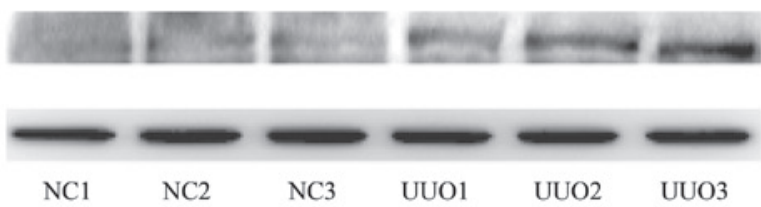

tubulin

UUO3

D

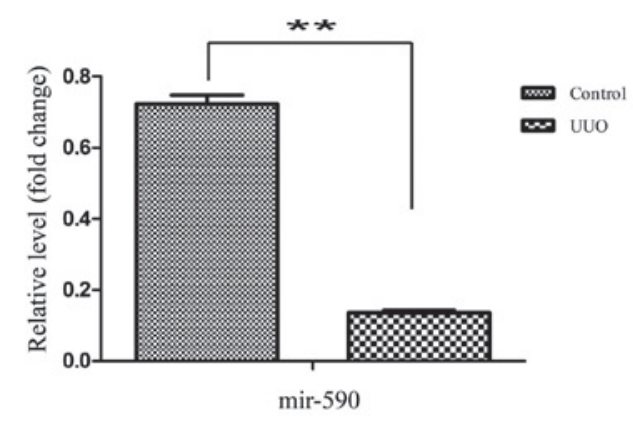

Figure 2. mRNA expression levels of miR-590 were decreased during EMT in vivo. (A) The EMT model with UUO kidneys. The UUO kidney section exhibited a large proportion of fibrotic tissues (revealed by the blue coloration). Magnification, x100. (B) Regulation of the mRNA expression levels of collagen, $\alpha$-SMA and E-cadherin in a kidney EMT model, as detected by RT-qPCR. (C) Regulation of the protein expression levels of laminin and E-cadherin in the kidney EMT model, as determined by western blot analysis. $\beta$-tubulin was used as a loading control. (D) Downregulation of the expression level of miR-590 in the kidney EMT model, as determined by RT-PCR. The results are shown as the fold change compared with the control. The data are expressed as the mean \pm standard deviation of three independent experiments. ${ }^{* *} \mathrm{P}<0.01$, compared with the control. a-SMA, $\alpha$-smooth muscle actin; UUO, unilateral ureteral obstruction.

consistently with the cell model, a decreased mRNA expression level of E-cadherin, and an increased mRNA expression level of collagen and $\alpha$-SMA, were observed in the RT-qPCR experiment (Fig. 2B). The western blot analysis revealed a decreased protein expression level of E-cadherin, and an increased protein expression level of laminin, in the UUO kidney (Fig. 2C). Similarly, the expression level of miR-590 was significantly decreased in the UUO animals compared with the control (Fig. 2D).

mir-590 downregulates TGF $\beta R 2$ by targeting its 3' UTR. Since a pronounced downregulation of miR-590 was revealed in the EMT model, to investigate the role exerted by miR-590 in EMT regulation, a search of the prediction software programs, TargetScan, miRanda and PicTar, was performed.It was observed that the 3 ' UTR of the TGF $\beta$ R2 mRNA contained a potential site for miR-590 (23). Subsequently, conservation of the 3' UTR of TGF $\beta$ R 2 was analyzed by blasting the sequences between different species, according to the University of California Santa Cruz genome database (Santa Cruz). As shown in Fig. 3A, the entire 3' UTR of TGFßR2 was 2,698 bp long, and was highly conserved among different species, including humans, rats, rabbits, shrews, tree shrews and dogs. Subsequently, the validation of the association between miR-590 and TGF $\beta R 2$ was assessed. Given that it was considered to be one of the most effective miRNAs in inhibiting the luciferase activity, the plasmid pCDNA-3.1-miR-590 was used in the present study. To examine whether miR-590 regulates TGF $\beta$ R 2 expression by directly binding to the predicted 3' UTR sequence, luciferase reporter plasmids containing the wild-type or the mutated binding site for miR-590 were constructed (Fig. 3B). These results revealed 


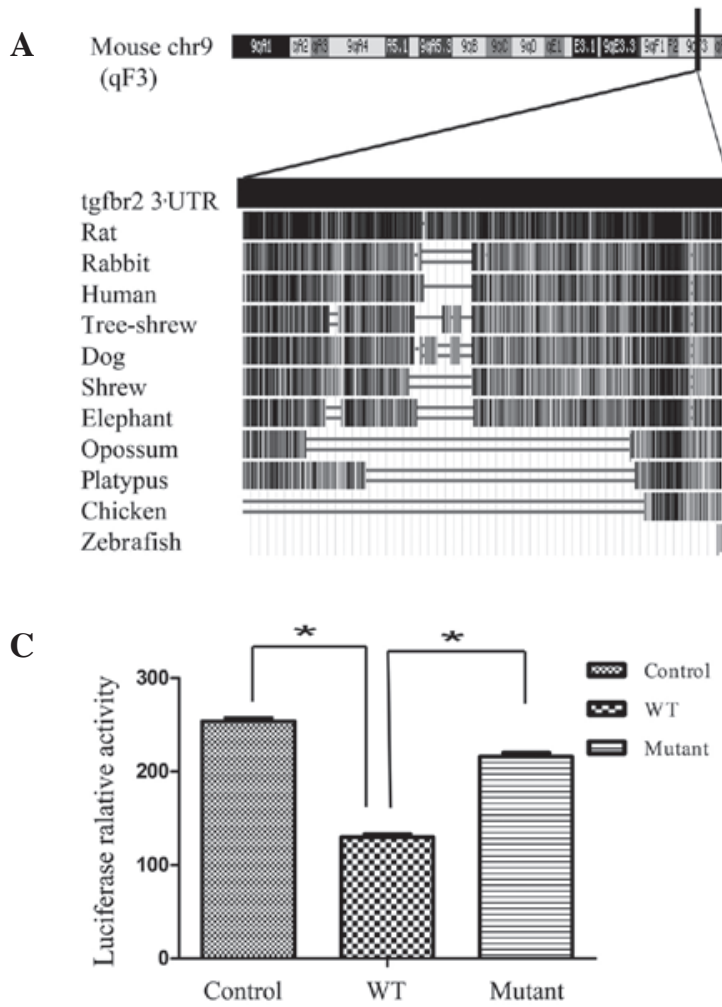

B

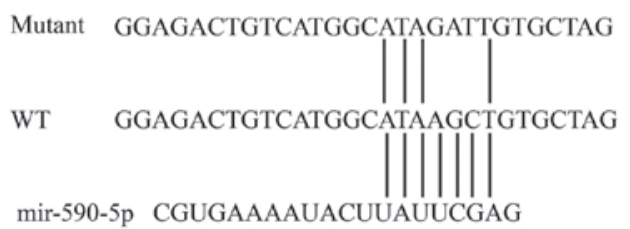

D

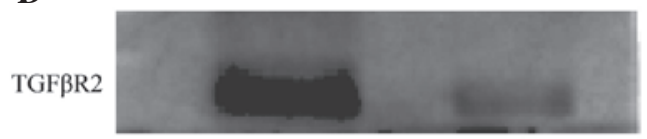

tubulin
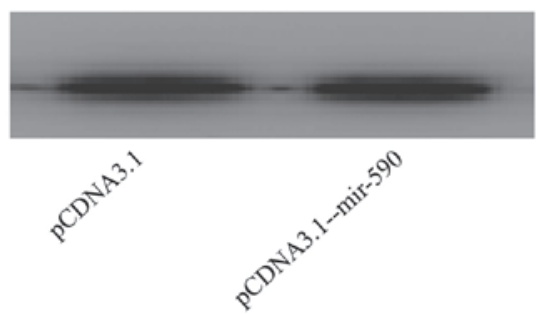

Figure 3. mir-590 downregulates TGF $\beta$ R2 by targeting its 3' UTR. (A) Bioinformatics analysis of the 3' UTR of TGF $\beta R 2$. The 3' UTR of TGF $\beta R 2$ is 2,698 bp in length, and is highly conserved among mammals, as revealed by the University of California, Santa Cruz genome database. (B and C) Analysis of the luciferase activity in HEK293T cells. HEK293T cells were transiently co-transfected with the luciferase reporter plasmid, containing the wild-type TGFBR2 3' UTR, or mutant variations, in the presence of pCDNA3.1-mir-590 or pCDNA3.1. (D) Effects of miR-590 on the endogenous TGFßR2 level in HK2 cells. The expression level was analyzed by western blotting. The data are presented as fold changes compared with the control cells. Data are presented as the mean \pm standard deviation, and are representative of at least three independent experiments. ${ }^{*} \mathrm{P}<0.05$, compared with the control. chr9, chromosome 9 ; WT, wild-type; UTR, untranslated region; TGF $\beta$ R2, transforming growth factor receptor $\beta 2$.

that the overexpression of miR-590 significantly suppressed the luciferase activity of the wild-type TGF $\beta$ R 2 3' UTR reporter, although not that of the mutant reporter containing a mutation in the miR-590-binding site (Fig. 3C). To examine the physiological importance of miR-590 in regulating TGF $\beta$ R2 production, the effect of miR-590 on TGF $\beta$ R 2 production in HK2 cells was assessed. The western blot analysis revealed that the transfection of HK2 cells with pCDNA-3.1-miR-590 markedly reduced the protein expression level of TGF 3 R2 (Fig. 3D). These results demonstrated that miR-590 is able to negatively regulate the expression of TGF $\beta$ R2 by directly targeting its $3^{\prime}$ UTR.

Effects of miR-590 and TGF $\beta R 2$ on EMT in HK2 cells. A consistent correlation between the expression levels of miR-590 and E-cadherin was observed, and a marked downregulation of miR-590 was identified in the EMT model. Furthermore, the expression of endogenous miR-590 was higher in HK2 cells compared with L929 cells (mice embryonic myofibroblasts; data not shown) indicating that the downregulation of miR-590 may explain phenotypic stabilization of mesenchymal features in the L929 cell line. To investigate the role of miR-590 in EMT, HK2 cells were transfected with pCDNA3.1-mir-590, and the expression level of mir-590 was significantly increased
(Fig. 4A). The induction of miR-590 markedly inhibited EMT in HK 2 cells, as revealed by epithelial marker changes. The RT-qPCR results demonstrated that the expression of the miR-590 plasmid in HK2 cells also resulted in a decrease in the mRNA expression of other known fibroblast markers, including $\alpha$-SMA and collagen I, and an increased mRNA expression of E-cadherin (Fig. 4B). Furthermore, the western blotting and immunofluorescence results revealed that transfection of the miR-590 plasmid markedly reduced the expression of laminin, and upregulated E-cadherin expression in HK2 cells (Fig. 4C and D). A previous study in our laboratory revealed that TGF $\beta 1$ induced EMT in vitro, accompanied by the upregulation of laminin, and the downregulation of E-cadherin, at the mRNA and protein levels in HK2 cells, whereas the transfection of exogenous miR-590 markedly attenuated the EMT process in HK2 cells.

The TGFßR2 3' UTR has a functional site for miR-590, and it is well known that TGF $\beta$ R 2 exerts an important role in the process of TGF $\beta$-mediated signal transduction by linking TGF $\beta 1$ to its receptor (TGF $\beta$ R2) and causing the activation of TGF $\beta$ receptor type I (TGF $\beta R 1$ ) kinase, leading to phosphorylation of the Smad2 and -3 complexes (24). To examine the effect of TGF $\beta$ R2 on EMT, TGF $\beta$ R2 siRNA or scrambled 
A

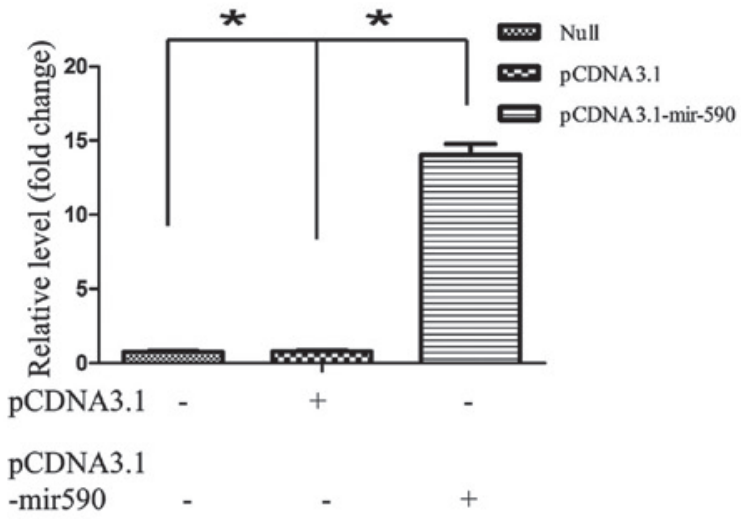

B

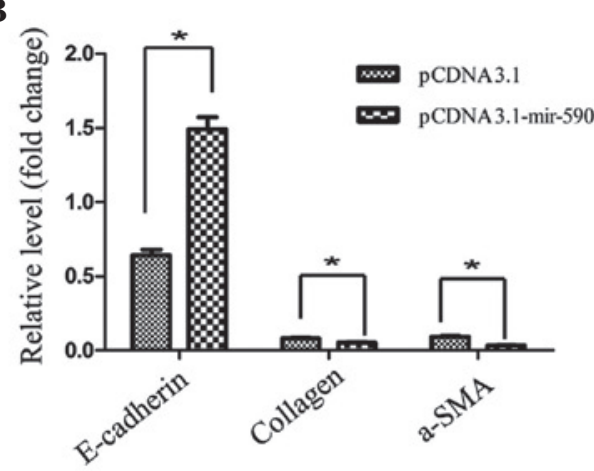

C

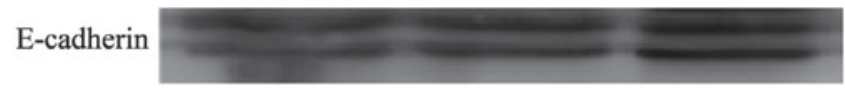

laminin

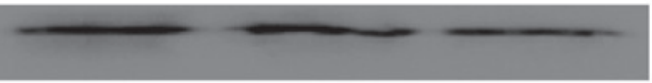

tubulin

pCDNA3.1

.1

pCDNA3.

$-\operatorname{mir} 590$

D

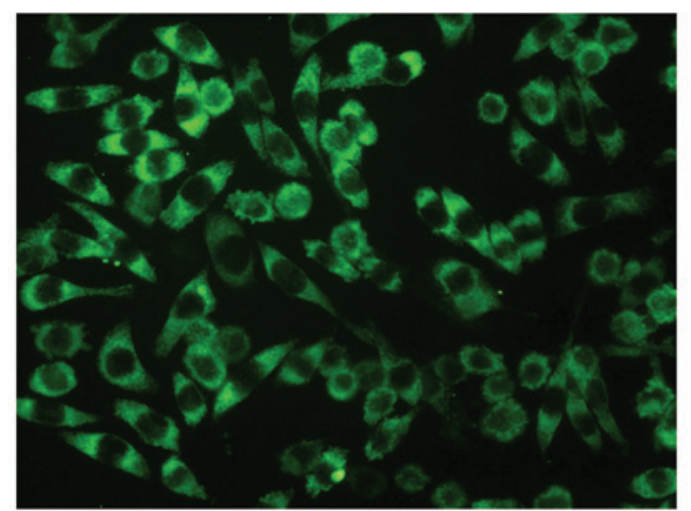

pCDNA3.1

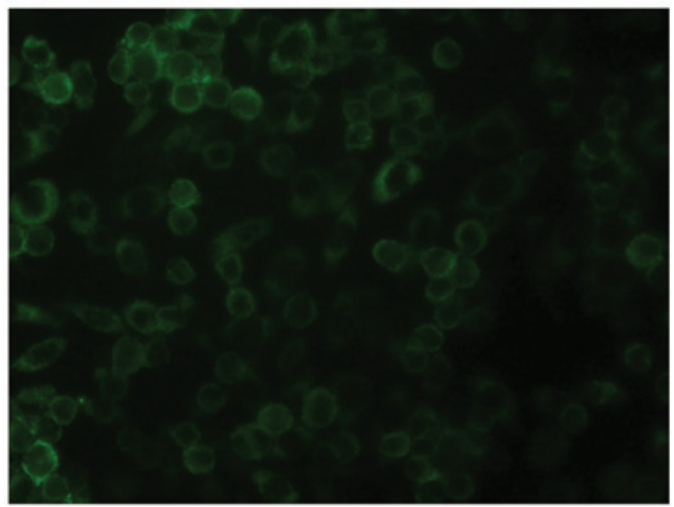

pCDNA3.1-mir-590

Figure 4. Effects of miR-590 on EMT in HK2 cells. (A) Validation of the transfection efficiency in HK2 cells with pCDNA3.1-mir-590 or pCDNA3.1. (B) The mRNA expression levels of E-cadherin, collagen and $\alpha$-SMA in HK2 cells $48 \mathrm{~h}$ following transfection with pCDNA3.1-mir-590 or pCDNA3.1. (C) The protein expression levels of laminin and E-cadherin $48 \mathrm{~h}$ following transfection with pCDNA3.1-mir-590 or pCDNA3.1. (D) The altered expression levels in HK2 cells following miR-590 overexpression, as measured by fluorescence. Magnification, $\mathrm{x} 400$. The data are shown as fold changes compared with control cells. The data are presented as the mean \pm standard deviation, and the results are representative of at least three independent experiments. "P $<0.05$, compared with the control. a-SMA, $\alpha$-smooth muscle actin.

siRNA was introduced into HK2 cells. As shown in Fig. 5A, TGF $\beta R 2$ siRNA effectively suppressed the protein levels of TGF $\beta$ R2, and prevent EMT in HK2 cells. The results revealed that E-cadherin expression was induced, whereas the mRNA expression levels of collagen and $\alpha$-SMA were reduced (Fig. 5B). HK2 cells transfected with TGF $\beta$ R2-specific siRNA exhibited increased protein levels of E-cadherin and a reduced protein expression of laminin, compared with the negative controls (Fig. 5C). By contrast, the upregulation of TGF $\beta$ R2 with pcmv-tag2b-TGF $\beta$ R2 in the HK2 cells was able to reverse the EMT-associated gene expression, as demonstrated by the increased protein expression levels of laminin, and reduced protein expression levels of E-cadherin, revealed in the western blot analysis of cells transfected with pcmv-tag2b-TGF $\beta$ R2 compared with transfected pcmv-tag2b vector (Fig. 5D).

\section{Discussion}

Regardless of the type of kidney disease, fibrosis is unavoidably the outcome, resulting in the marked destruction of the kidney structure, with consequential functional deterioration (24). Myofibroblasts contribute to the accumulation of the extracellular matrix observed in fibrotic disease, and it was demonstrated that myofibroblasts may originate from renal residential fibro- 
A

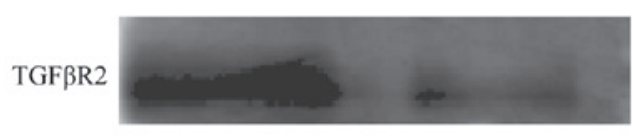

tubulin
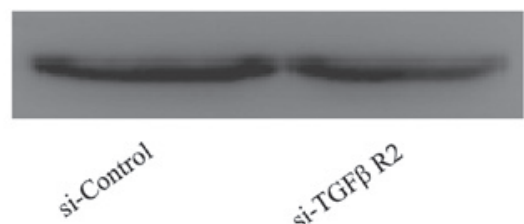

C

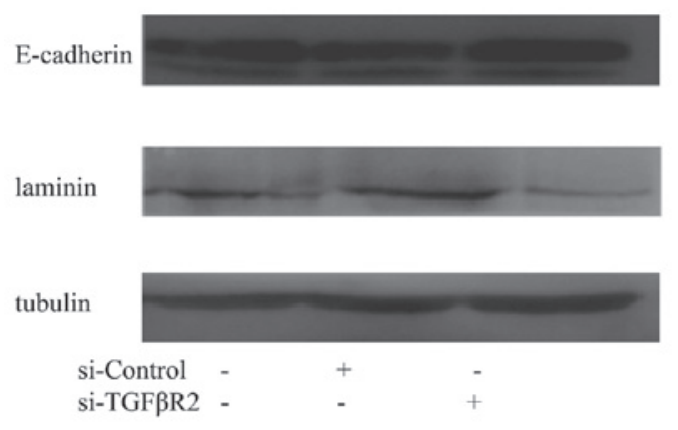

B

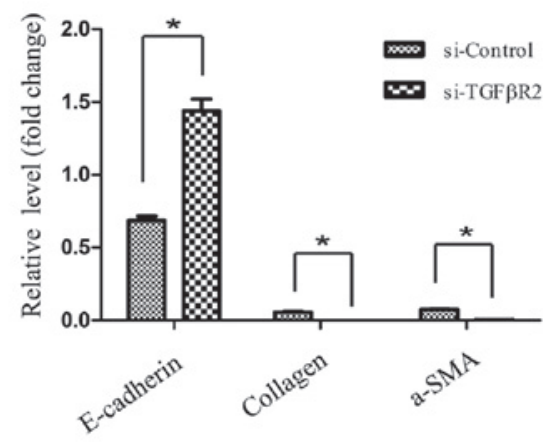

D

E-cadherin

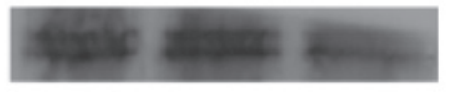

laminin

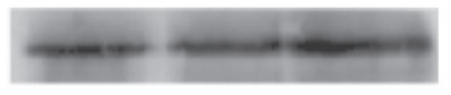

TGF $\beta$ R2

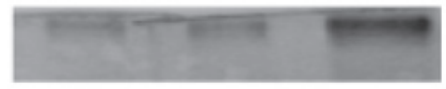

tubulin

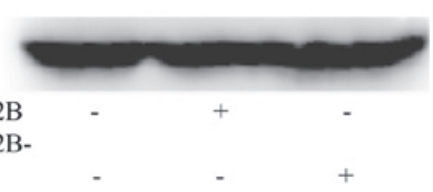

Figure 5. Effect of TGF $\beta$ R2 on EMT. (A) The expression of TGF $\beta$ R2 in HK2 cells was successfully suppressed by TGF $\beta R 2$ siRNA. (B) The relative mRNA levels of E-cadherin, collagen and $\alpha$-SMA in HK2 cells, following TGF $\beta$ R2 siRNA treatment for $48 \mathrm{~h}$. (C) Western blot analysis of the alterations of E-cadherin and laminin in HK2 cells treated with TGF $\beta$ R2 siRNA for 48 h. (D) The overexpression of TGF $\beta$ R2 with pCMV-tag2B-TGF $\beta$ R2 promoted EMT in HK2 cells. The data are shown as the fold changes compared with cells transfected with the control. The data are presented as the mean \pm standard deviation of three independent experiments. $\mathrm{P}<0.05$, compared with the control. TGF $\beta \mathrm{R} 2$, transforming growth factor receptor $\beta 2$; a-SMA, $\alpha$-smooth muscle actin.

blasts $(25,26)$. In addition to the residential fibroblasts, other sources of fibroblasts have been studied, including fibrocytes and pericytes. The EMT of mature tubular epithelial cells is another source of fibroblasts, which are fundamentally linked to the process of renal fibrosis $(27,28)$. As revealed in the present study, the mRNA expression level of E-cadherin in the epithelial cells was markedly decreased, whereas the mesenchymal markers, collagen I and $\alpha$-SMA, were increased in the EMT model in vitro and in vivo. Consistent with the results of the RT-qPCR analysis, the western blotting data further confirmed that the EMT process existed in TGF 31 -treated HK2 cells and the UUO model, as demonstrated by the lower expression levels of E-cadherin and higher expression levels of laminin. Furthermore, previous studies revealed that a proportion of the fibroblasts, which are predominantly the functional cells in this process, originate from the tubular epithelial cells in the diseased kidney by EMT (28-30), which has an important role in the process of kidney disease. Therefore, understanding the regulatory mechanisms of EMT may help to elucidate the events involved in the process of fibrosis, leading to the development of novel therapeutics for treating kidney disease.

miRNAs are endogenously encoded, non-coding RNAs which predominantly post-transcriptionally regulate gene expression through either translational suppressors or mRNA degradation (8). miRNAs, a class of newly identified regulators, were demonstrated to be involved in the process of EMT. In vitro and animal studies revealed that these miRNAs (miR-200a,
miR-200b and miR-205) suppress EMT by targeting ZEB1 and ZEB2 (11-13). Other studies demonstrated that miR-655 and miR-34a are associated with EMT-suppressive miRNAs $(31,32)$. The present study has revealed that miR-590 causes EMT, as demonstrated by the evidence that, to the best of our knowledge, for the first time, miR-590 affects EMT in HK2 cells. Transfection of the HK2 cells with pCDNA3.1-mir-590 resulted in an inhibition of a number of the fibrotic changes, and the promotion of various epithelial characteristics. Therefore, miR-590 appears to exert a key role in EMT, and may promote fibrosis.

Previous studies revealed that miR-590 has multiple, experimentally validated target genes, including TGF $\beta$ R2, protein polybromo-1, S100A10 and the cell adhesion molecule L1-like gene (33-36). Among these targets, TGF $\beta R 2$ was revealed to be associated with EMT in epithelial cells. To investigate the mechanism underlying the involvement of miR-590 in EMT, the present study has revealed that miR590 directly targets TGF $\beta R 2$, as demonstrated by the luciferase reporter activity assays and western blot analyses. Protein levels of TGF $\beta$ R2 were markedly decreased following miR-590 overexpression. Additionally, E-cadherin expression was upregulated and the expression of laminin was decreased. TGF $\beta$ R2 was identified as a functional target of miR-590, and this target is well established as a component of the TGF $\beta$ signaling pathway. The TGF $\beta$ signaling pathway consists of TGF $\beta$, TGF $\beta$, the Smad protein family and several other important transcriptional regulatory factors. The TGF $\beta \mathrm{R}$ family comprises three 
subtypes, TGF $\beta R 1$, TGF $\beta$ R 2 and TGF $\beta$ R3, although only TGF $\beta R 2$ may accept TGF $\beta 1$ as a protein partner independently of the other two receptors, which are unable to function in the absence of TGF $\beta R 2$ (37). Furthermore, a previous study demonstrated that mir-655 is an EMT-suppressive miRNA, which targets TGF $\beta$ R2 (32). In the present study, it was also revealed that the knockdown of TGF $\beta$ R 2 with siRNA elicited a similar pattern of alteration of the EMT-associated genes, whereas the transfection of pcmv-tag2B-TGF $\beta$ R2 reversed the protein expression, results which are consistent with the previous hypothesis. Therefore, mir-590 was identified as an EMT-suppressive miRNA in the present study.

In conclusion, the results reported in the present study provide the first evidence, to the best of our knowledge, that miR-590 is involved in the process of EMT in tubular epithelial cells. The overexpression of miR-590 markedly increased E-cadherin expression and suppressed laminin expression in HK2 cells, clearly indicating that this miRNA is an inhibitor of EMT. Furthermore, TGF $\beta$ R2, which is a core component of the TGF $\beta$ signaling pathway, was characterized as a direct target of miR-590. The present results suggest that the EMT-suppressor miR-590, targeting TGF $\beta$ R2, has the potential to serve as a biomarker of, and a potential therapeutic target for, fibrosis.

\section{Acknowledgements}

This study was supported by the National Natural Science Foundation of China(no.31271563), the National Basic Research Program of China (no. 2011CB944002) and the Chongqing Application Development Project (no. cstc2014yykfB10003).

\section{References}

1. Kalluri R and Weinberg RA: The basics of epithelial-mesenchymal transition. J Clin Invest 119: 1420-1428, 2009.

2. Liu Y: Epithelial to mesenchymal transition in renal fibrogenesis: Pathologic significance, molecular mechanism and therapeutic intervention. J Am Soc Nephrol 15: 1-12, 2004.

3. Schrimpf C and Duffield JS: Mechanisms of fibrosis: the role of the pericyte. Curr Opin Nephrol Hypertens 20: 297-305, 2011.

4. Lin SL, Kisseleva T, Brenner DA and Duffield JS: Pericytes and perivascular fibroblasts are the primary source of collagenproducing cell sin obstructive fibrosis of the kidney. Am J Pathol 173: 1617-1627, 2008.

5. Humphreys BD, Lin SL, Kobayashi A, Hudson TE, Nowlin BT, and Bonventre JV Valerius MT, McMahon AP and Duffield JS: Fate tracing reveals the pericyte and not epithelial origin of myofibroblasts in kidney fibrosis. Am J Pathol 176: 85-97, 2010.

6. Thiery JP, Acloque H, Huang RY and Nieto MA: Epithelial-mesenchymal transitions in development and disease. Cell 139: 871-890, 2009.

7. Thiery JP: Epithelial-mesenchymal transitions in development and pathologies. Curr Opin Cell Biol 15: 740-746, 2003.

8. He L and Hannon GJ: MicroRNAs: Small RNAs with a big role in gene regulation. Nat Rev Genet 5: 522-531, 2004.

9. Zhang J and Ma L: MicroRNA control of epithelial-mesenchymal transition and metastasis. Cancer Metastasis Rev 31: 653-662, 2012

10. Akkina $\mathrm{S}$ and Becker BN: MicroRNAs in kidney function and disease. Transl Res 157: 236-240, 2011.

11. Gregory PA, Bert AG, Paterson EL, Barry SC, Tsykin A, Farshid G Vadas MA, Khew-Goodall Y and Goodall GJ: The miR-200 family and miR-205 regulate epithelial to mesenchymal transition by targeting ZEB1 and SIP1. Nat Cell Biol 10: 593-601, 2008.

12. Paterson EL, Kolesnikoff N, Gregory PA, Bert AG, Khew-Goodall Y and Goodall GJ: The microRNA-200 family regulates epithelial to mesenchymal transition. Scientific World Journal 8: 901-904, 2008.

13. Korpal M,Lee ES, Hu G and Kang Y: The miR-200 family inhibits epithelial-mesenchymal transition and cancer cell migration by direct targeting of E-cadherin transcriptional repressors ZEB1 and ZEB2. J Biol Chem 283: 14910-14914, 2008.
14. Kong W, Yang H, He L, Zhao JJ, Coppola D, Dalton WS and Cheng JQ: MicroRNA-155 is regulated by the transforming growth factor beta/Smad pathway and contributes to epithelial cell plasticity by targeting RhoA. Mol Cell Biol 28: 6773-6784, 2008.

15. Chen YT, Chang FC, Wu CF, Chou YH, Hsu HL, Chiang WC, Shen J, Chen YM, Wu KD, Tsai TJ, Duffield JS and Lin SL: Platelet-derived growth factor receptor signaling activates pericyte myofibroblast transition in obstructive and post-ischemic kidney fibrosis. Kidney Int. 80: 1170-1181, 2011.

16. Yang J, Shultz RW, Mars WM, Wegner RE, Li Y, Dai C, Nejak K and Liu Y: Disruption of tissue type plasminogen activator gene in mice reduces renal interstitial fibrosis in obstructive nephropathy. J Clin Invest 110: 1525-1538, 2002

17. Morales C, González GE, Rodríguez M, Bertolasi CA and Gelpi RJ: Histopathologic time course of myocardial infarct in rabbit hearts. Cardiovasc Pathol 11: 339-345, 2002.

18. Junqueira LC, Bignolas G and Brentani RR: Picrosirius staining plus polarization microscopy, a specific method for collagen detection in tissue sections. Histochem J 11: 447-455, 1979.

19. Chen L, Faulhaber-Walter R, Wen Y, Huang Y, Mizel D, Chen M, Sequeira Lopez ML, Weinstein LS, Gomez RA, Briggs JP and Schnermann J: Renal failure in mice with Gsalpha deletion in juxtaglomerular cells. Am J Nephrol. 32: 83-94, 2010.

20. Kaimori A, Potter J, Kaimori JY, Wang C, Mezey E and Koteish A: Transforming growth factor-beta 1 induces an epithelial- to -mesenchymal transition state in mouse hepatocytes in vitro. J Biol Chem 282: 22089-22101, 2007.

21. Aresu L, Rastaldi MP, Scanziani E, Baily J, Radaelli E, Pregel P and Valenza F: Epithelial-mesenchymal transition (EMT) of renal tubular cells in canine glomerulonephritis. Virchows Arch 451: 937-942, 2007.

22. Huang XR, Chung AC, Wang XJ, Lai KN and Lan HY: Mice overexpressing latent TGF-beta 1 are protected against renal fibrosis in obstructive kidney disease. Am J Physiol Renal Physiol 295: F118-F127, 2008.

23. Zhang J, Zhang H, Liu J, Tu X, Zang Y, Zhu J, Chen J, Dong L and Zhang J: MiR-30 inhibits TGF-bl-induced epithelial-to-mesenchymal transition in hepatocyte by targeting Snaill. Biochem Biophys Res Commun 417: 1100-1105, 2012.

24. Liu Y: Cellular and molecular mechanisms of renal fibrosis. Nat Rev Nephrol 7: 684-696, 2011.

25 . Hills CE and Squires PE: The role of TGF- $\beta$ and epithelial- to mesenchymal transition in diabetic nephropathy. Cytokine Growth Factor Rev 22: 131-139, 2011.

26. Barnes JL and Gorin Y: Myofibroblast differentiation during fibrosis: Role of NAD(P)H oxidases. Kidney Int 79: 944-956, 2011.

27. Lan HY: Tubular epithelial-myofibroblast transdifferentiation mechanisms in proximal tubule cells. Curr Opin Nephrol Hypertens 12: 25-29, 2003.

28. Zeisberg M and Duffield JS: Resolved: EMT produces fibroblasts in the kidney. J Am Soc Nephrol 21: 1247-1253, 2010.

29. Yang J and Liu Y: Blockage of tubular epithelial to myofibroblast transition by hepatocyte growth factor prevents renal interstitial fibrosis. J Am Soc Nephrol 13: 96-107, 2002.

30. Iwano M: EMT and TGF-beta in renal fibrosis. Front Biosci (Schol Ed) 2: 229-238, 2010.

31. Du R, Sun W, Xia L, Zhao A, Yu Y, Zhao L, Wang H, Huang C and Sun S: Hypoxia-induced down-regulation of microRNA-34a promotes EMT by targeting the Notch signaling pathway in tubular epithelial cells. PLoS One 7: e30771, 2012.

32. Harazono Y, Muramatsu T, Endo H, Uzawa N, Kawano T, Harada K, Inazawa J and Kozaki K: MiR-655 is an EMTsuppressive microRNA targeting ZEB1 and TGFBR2. PLoS One 8: e62757, 2013.

33. Chu Y, Ouyang Y, Wang F, Zheng A, Bai L, Han L, Chen Y and Wang H: MicroRNA-590 promotes cervical cancer cell growth and invasion by targeting CHL1. J Cell Biochem 115: 847-853, 2014.

34. Xiao X, Tang C, Xiao S, Fu C and Yu P: Enhancement of proliferation and invasion by MicroRNA-590-5p via targeting PBRM1 in clear cell renal carcinoma cells. Oncol Res 20: 537-544, 2013.

35. Shan X, Miao Y, Fan R, Qian H, Chen P, Liu H, Yan X, Li J and Zhou F: MiR-590-5P inhibits growth of HepG2 cells via decrease of S100A10 expression and inhibition of the Wnt pathway. Int J Mol Sci 14: 8556-8569, 2013.

36. Jiang X, Xiang G, Wang Y, Zhang L, Yang X, Cao L, Peng H, Xue $P$ and Chen D: MicroRNA-590-5p regulates proliferation and invasion in human hepatocellular carcinoma cells by targeting TGF- $\beta$ RII. Mol Cells 33: 545-551, 2012.

37. Shi Y and Massagué J: Mechanisms of TGF-beta signaling from cell membrane to the nucleus. Cell 113: 685-700, 2003. 